\title{
o Idiopathic Middle Meningeal Arteriovenous Case Report Fistula Associated with Segmental Arterial Mediolysis: A Case Report
}

Masahiro Uchimura, ${ }^{1}$ Haruo Takigawa, ${ }^{1}$ Tsutomu Yoshikane,${ }^{2}$ and Yuichi Anno $^{1}$

Objective: Middle meningeal arteriovenous fistula (MMAVF) is typically post-traumatic or iatrogenic in origin, but it can have an idiopathic origin in rare cases. Here, we report a case of idiopathic MMAVF complicated by segmental arterial mediolysis (SAM).

Case Presentation: The patient was a 63-year-old woman admitted to our hospital with symptoms of right pulsating tinnitus for the past 2 months. She had no significant medical history. MRI and DSA revealed a right MMAVF. Selective embolization using coils was performed. Seven years later, when she underwent laparotomy for intestinal perforation, multiple aneurysms were found in the abdominal cavity. Left hemicolectomy was performed, and an aneurysm of the gastroepiploic artery was resected. Based on a pathological examination of the aneurysm, the patient was diagnosed with SAM. Coil embolization was performed to prevent rupture.

Conclusion: This case report shows that MMAVF may be associated with SAM. Fistula embolization with coils is an effective treatment of MMAVF associated with SAM. Therefore, it is important to check for systemic diseases, such as SAM when idiopathic MMAVF is detected.

Keywords \ middle meningeal arteriovenous fistulas, segmental arterial mediolysis, endovascular treatment

\section{Introduction}

Middle meningeal arteriovenous fistula (MMAVF) is relatively rare, and most of the reported cases have a traumatic or iatrogenic origin. However, rare cases with an idiopathic origin have also been reported. ${ }^{1-6)}$ MMAVF is suspected based on symptoms, such as tinnitus, bruit, exophthalmos, and findings of dilated vessels on MRI, and is diagnosed using DSA. ${ }^{5,6)}$ Segmental arterial mediolysis (SAM) is a rare vasculopathy characterized by an unusual noninflammatory

${ }^{1}$ Department of Neurosurgery, Matsue City Hospital, Matsue, Shimane, Japan

${ }^{2}$ Department of Neurosurgery, Shimane University Faculty of Medicine, Izumo, Shimane, Japan

Received: February 25, 2021; Accepted: April 30, 2021 Corresponding author: Masahiro Uchimura. Department of Neurosurgery, Matsue City Hospital, 32-1, Noshirocho, Matsue, Shimane 690-8509, Japan

Email: muchimura8953@gmail.com

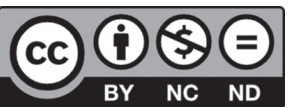

This work is licensed under a Creative Commons Attribution-NonCommercialNoDerivatives International License.

(C)2021 The Japanese Society for Neuroendovascular Therapy and nonatherosclerotic disorder of the arterial medial layer. ${ }^{7-9)}$ SAM usually affects the abdominal visceral arteries and forms aneurysms, but any vessel may be involved, including those in the head and neck region. ${ }^{7)}$ In this case, idiopathic MMAVF was initially diagnosed and treated by selective embolization with coils. Seven years later, SAM was diagnosed histopathologically from aneurysms of the abdominal splanchnic arteries. This is the first case report showing the association between MMAVF and SAM and its effective treatment by fistula embolization with coils.

\section{Case Presentation}

A 63-year-old woman without any medical history, including trauma, was referred to our hospital for a right pulsating tinnitus for 2 months. Neurologically, no other abnormalities were found. MRA revealed a dilated vessel in the right middle skull base near the foramen spinosum (Fig. 1A); thus, an arteriovenous fistula was suspected. CT revealed no skull fracture around the dilated vessel (Fig. 1B).

MRA also revealed the fenestration of the right cervical internal carotid artery (ICA) and the fusiform aneurysm in left cervical ICA(Fig. 1C). Cerebral angiography 


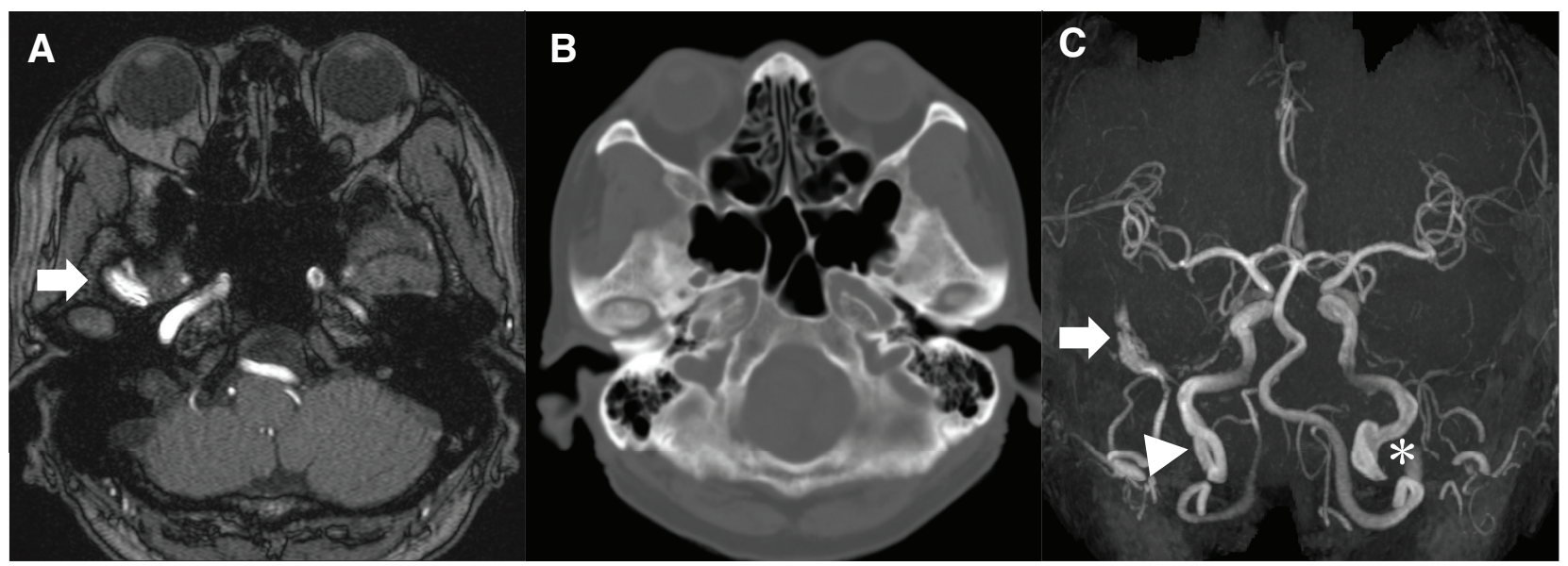

Fig. 1 Initial MRA and CT of the brain. (A) Initial MRA reveals a dilated vessel (arrow) in the right middle skull base near the foramen spinosum. (B) CT reveals no skull fracture around the dilated vessel.

was performed, and right external carotid arteriography revealed a high flow dural arteriovenous fistula of the right middle meningeal artery (MMA) branch with drainage into the middle meningeal vein (MMV) (Fig. 2A and 2B). The MMV drains into the inferior petrosal sinus and pterygoid plexus via the ipsilateral cavernous sinus. Retrograde drainage into the cortical veins was not revealed (Fig. 2C). The fistula was located in the anterior branch of the MMA immediately after the foramen spinosum (Fig. 2D).

\section{Endovascular treatment}

The right MMAVF was scheduled to be embolized with coils. Oral administration of aspirin $(100 \mathrm{mg})$ was started 5 days before the treatment to prevent periprocedural thromboembolism. Under local anesthesia, a 6-Fr sheath was inserted into the right femoral artery, and a 6-Fr 100-cm ENVOY (Johnson \& Johnson, New Brunswick, NJ, USA) was navigated to the right external carotid artery. From the right external carotid artery, the Renegade (Boston Scientific, Marlborough, MA, USA) was guided to the MMA using a Radifocus guidewire 14 (Terumo, Tokyo, Japan). Selective angiography of the MMA revealed a fistula located in the anterior branch of the MMA just after the foramen spinosum, and the Renegade was inserted into the venous side via the fistula by pulling it from the distal anterior branch of the MMA (Fig. 2E and 2G). The venous side in contact with the fistula was occluded by inserting five GDC-10 ultrasoft coils (Boston Scientific). Complete occlusion was achieved for only the venous side that was in contact with the fistula, and the MMA was preserved by final angiography (Fig. 2F and $\mathbf{2 H}$ ).
(C) MRA reveals the AVF (arrow), fenestration of the right cervical ICA (arrowhead), and the fusiform aneurysm in the left cervical ICA (*). AVF: arteriovenous fistula; ICA: internal carotid artery

\section{Postoperative course}

Postoperatively, the right pulsating tinnitus disappeared, and no de novo neurological abnormalities were observed. The patient was discharged 4 days post-treatment. Onemonth post-treatment, MRA revealed that the dilated vessel in the right middle skull base had disappeared.

Seven years later, the patient was referred for severe stomachache. Contrast-enhanced CT revealed stenosis of the sigmoid colon with pneumoperitoneum. CTA revealed vascular dilation of the common hepatic, gastroepiploic, dorsal splenic, and left gastric arteries (Fig. 3A). Left hemicolectomy was performed, and the aneurysm of the gastroepiploic artery was resected. Pathological examination of the aneurysm revealed mediolysis and separation; however, the medial layer showed no evidence of inflammation (Fig. 3B and 3C). Based on these findings, the patient was diagnosed with SAM. Thus, coil embolization was scheduled to prevent rupture. Head MRI revealed that the existing fistula had disappeared (Fig. 4A). Additionally, the fenestration of the right cervical ICA and the fusiform aneurysm in the left cervical ICA did not change, and new fistula or aneurysm was not observed (Fig. 4B).

\section{Discussion}

This case report highlighted two important clinical issues. First, MMAVF may be associated with SAM. Second, fistula embolization with coils is effective in treating MMAVF associated with SAM.

MMAVF is a relatively rare disease, with typically post-traumatic or iatrogenic origins, ${ }^{1-6)}$ but rare idiopathic cases have also been reported. ${ }^{2,4)}$ A previous report has 


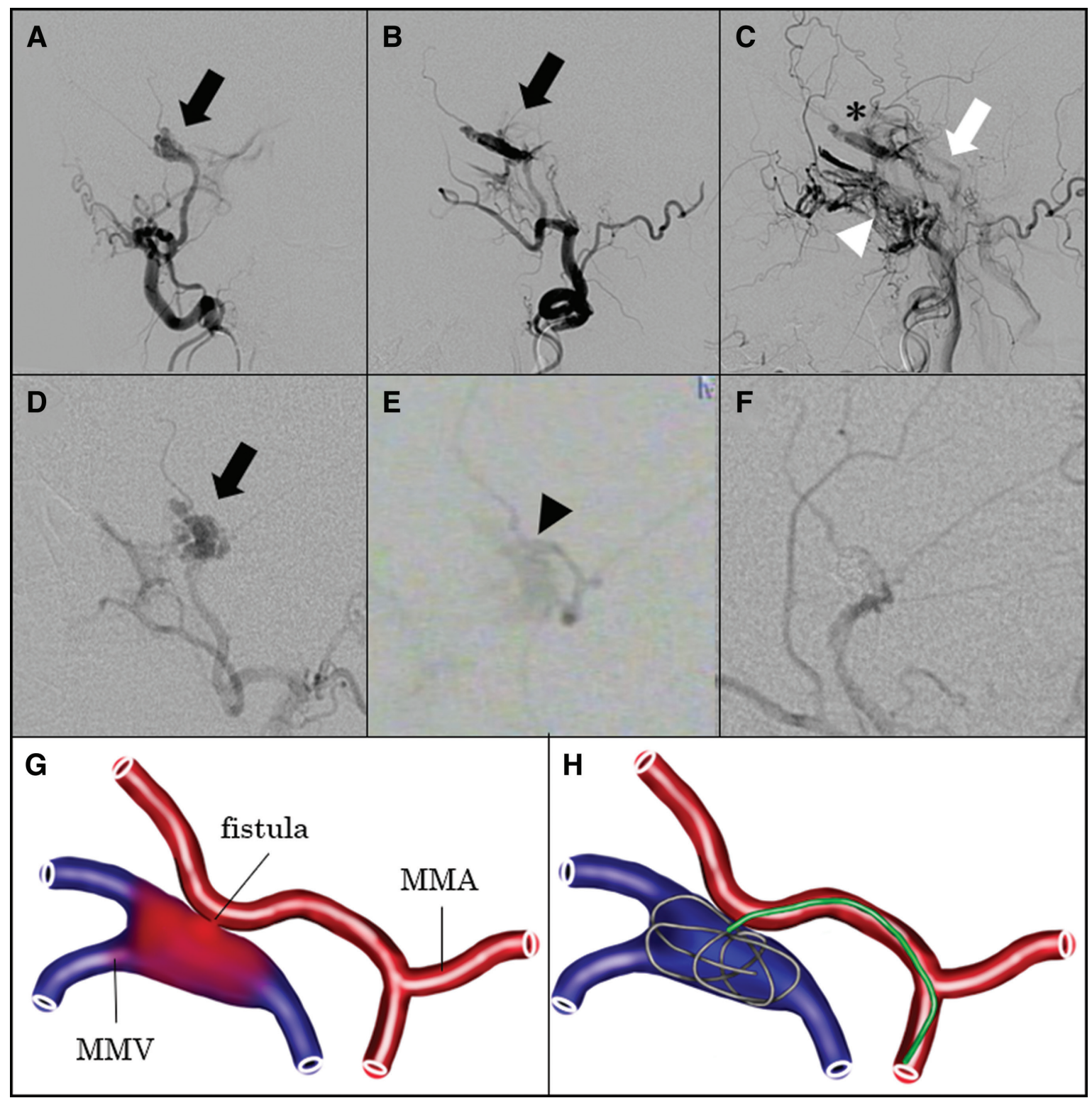

Fig. 2 DSA and schema of MMAVF. (A) Anteroposterior and (B, C) lateral views of the right external carotid angiogram show the AVF (black arrows) supplied by the dilated right MMA, draining into the cavernous sinus (*), inferior petrosal sinus (white arrow), and pterygoid plexus (white arrowhead). (D) A working angle view of the angiogram demonstrates the dilation of the MMA immediately after the foramen spinosum (black allow). (E) Superselective angiography from a microcatheter inserted into the MMA reveals the fistula located in the anterior branch of the MMA just after the foramen spinosum (black arrowhead). (F) A working angle view of the right external carotid angiogram after embolization shows the disappearance of the fistula and preservation of the MMA. (G) The schema shows a dural AVF of MMA branch with drainage into the MMV. (H) The schema shows a microcatheter is inserted into the venous side via the fistula and the venous side in contact with the fistula is occluded by inserting coils. AVF: arteriovenous fistula; MMA: middle meningeal artery; MMAVF: middle meningeal arteriovenous fistula; MMV: middle meningeal vein

shown that most cases of traumatic MMAVF occurred due to a tear in the artery wall caused by a skull fracture. ${ }^{1,5)}$ Moreover, separation of the dura mater from the bone can cause a traumatic MMAVF without skull fracture. ${ }^{10)}$ Contrastingly, the underlying causes for idiopathic MMAVF remain unclear. In this case, idiopathic MMAVF was initially diagnosed, and 7 years later, SAM was diagnosed, which we believe was associated with MMAVF. To the best of our knowledge, this is the first reported case of MMAVF complicated by SAM.

SAM was first reported in 1976 by Slavin et al. ${ }^{11)}$ It is a rare vasculopathy characterized by an unusual noninflammatory and nonatherosclerotic disorder of the arterial medial layer. ${ }^{7-9)}$ It typically affects the abdominal visceral splanchnic arteries, and the most common clinical presentation is abdominal pain or shock. However, any 


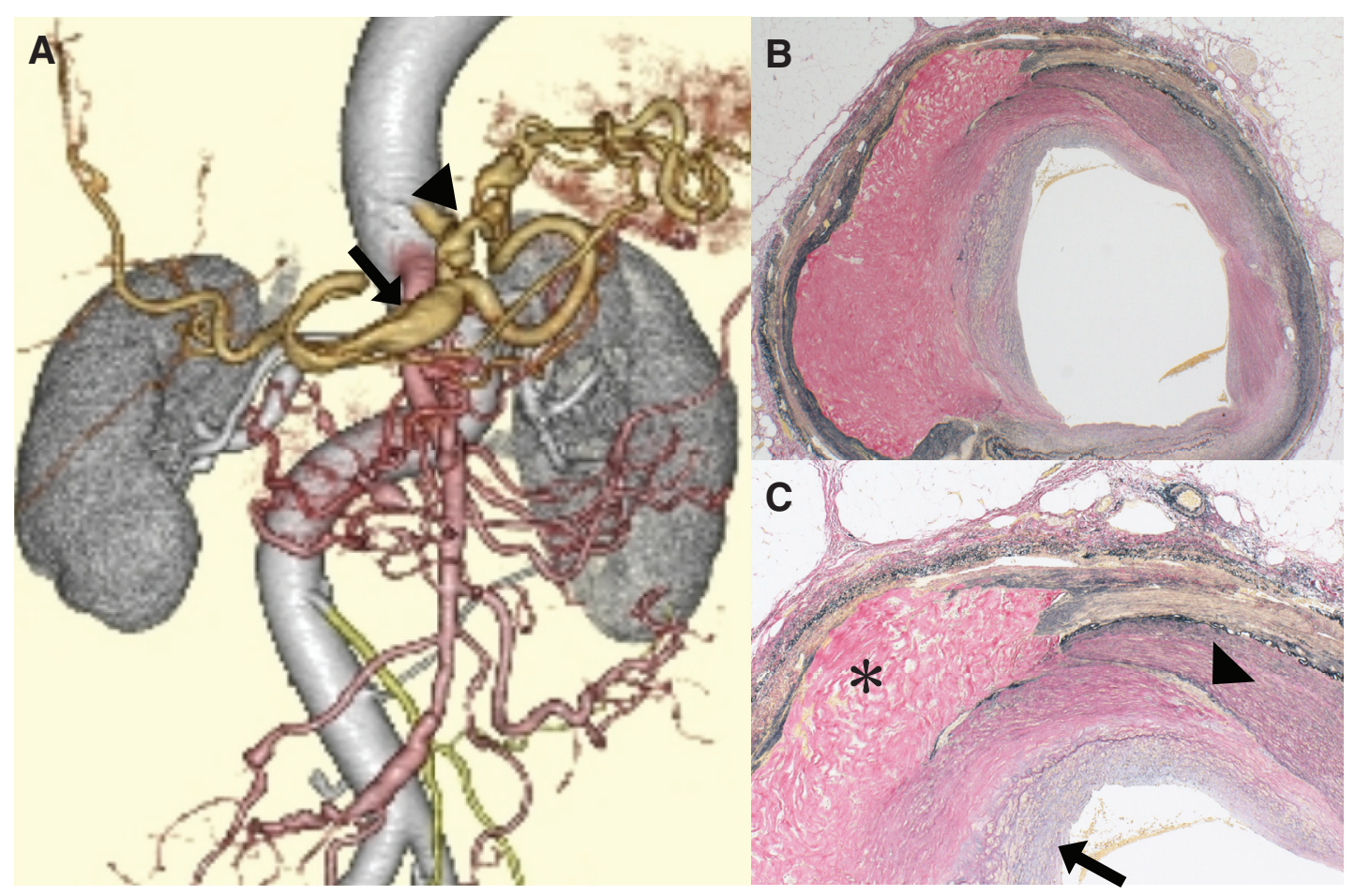

Fig. 3 Contrast-enhanced abdominal CT and the pathological findings of the aneurysm. (A) 3D CTA reveals multiple aneurysms at the common hepatic (arrow), left gastric (arrowhead), gastroepiploic, and dorsal splenic arteries. (B, C) Pathological examination of the aneurysm reveals the mediolysis $(*)$ and separation of the medial layer (arrowhead) and fibrous thickening of the intima (arrow), but evidence of inflammation is not observed.

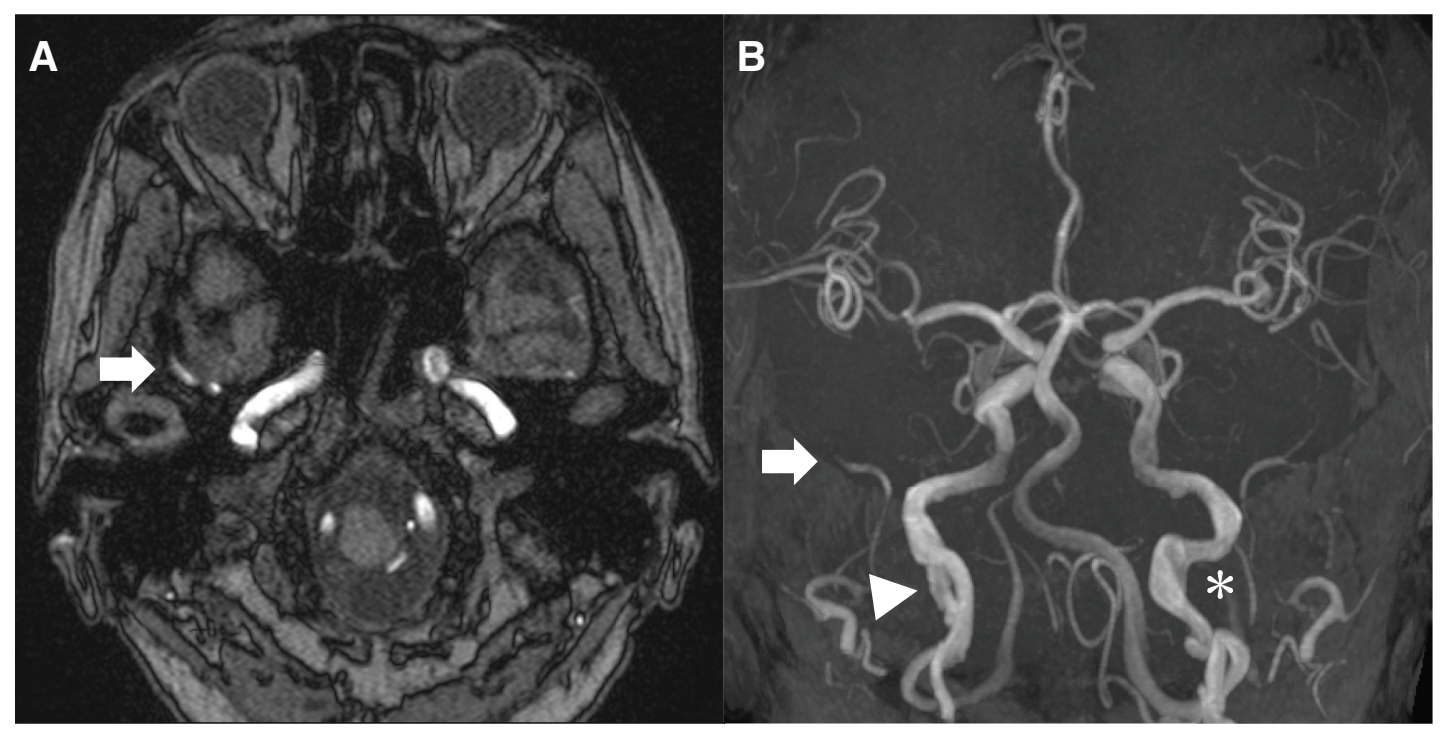

Fig. 4 MRA of the brain 7 years after the treatment. (A) MRA reveals the disappearance of the fistula (arrow). No new fistula or aneurysm is observed. (B) MRA shows that the AVF disappeared (arrow), and the fenestration of the right cervical ICA (arrowhead) and the fusiform aneurysm in the left cervical ICA $(*)$ do not change. AVF: arteriovenous fistula; ICA: internal carotid artery

vessel may be involved, including those in the head and neck region. ${ }^{7)}$ Although the diagnostic frequency of SAM has increased with the recent advancements in imaging modalities, an accurate diagnosis is still difficult. The differential diagnoses for SAM include fibromuscular dysplasia (FMD), connective tissue diseases (e.g., polyarteritis nodosa, antineutrophil cytoplasmic antibodiesassociated vasculitis, giant cell arteritis, Behcet's disease, 
and Kawasaki disease), inherited diseases (e.g., type IV Ehlers-Danlos syndrome, Marfan syndrome, and neurofibromatosis), and atherosclerosis. ${ }^{8)}$ The gold standard method to determine the differential diagnoses between these vascular diseases is histopathology. ${ }^{8,9}$ Characteristic pathological findings of SAM include mediolysis, separation of the outer media, and formation of arterial gaps. $^{7-9)}$ FMD and SAM are noninflammatory and nonatherosclerotic arterial diseases that need to be diagnosed urgently, particularly because they could affect various organs with fatal outcomes. ${ }^{7-9)}$

Closure of the fistula with coils is an effective treatment for MMAVF complicated by SAM. Although spontaneous closure of a traumatic shunt has been reported in previous reports, early treatment should be considered due to the risk of intracranial hemorrhage, particularly in cases with cortical venous drainage. ${ }^{2)}$ In symptomatic cases, early treatment is desirable before the fistula increases and treatment becomes more difficult. ${ }^{12)}$ Although the natural history of idiopathic MMAVF remains unclear because of its rarity, it is thought that non-traumatic lesions pose a risk of intracranial hemorrhage regardless of their etiology. ${ }^{2)}$ MMAVF has traditionally been treated surgically, but more recently, embolization has been reported to be a relatively easy and safe initial treatment. ${ }^{13)}$ Previous reports have shown that transarterial embolization with coils or liquid embolic agents is effective for the management of traumatic MMAVF.5,6,13,14) Proximal occlusion is ineffective, and it is necessary to block the fistulous portion securely. ${ }^{13,14)}$ It is unknown if non-traumatic MMAVF typically has a single shunt and whether transarterial embolization is effective. However, in this case, it was confirmed that the AVF was due to a single shunt from the MMA, and the fistula disappeared following transarterial selective embolization using coils. Therefore, if a single shunt can be confirmed and a microcatheter can be introduced into the draining veins via the fistula, selective embolization by coils is considered an effective treatment even for MMAVF associated with SAM. However, since the possibility of future recurrence of MMAVF associated with SAM cannot be ruled out due to the fragility of the vessel, it may have been better to embolize the entire diseased MMA, including the fistula with coils in this case. Furthermore, although no consensus has been established on the best endovascular approach to closure of the carotid-cavernous fistulas caused by an Ehlers-Danlos Syndrome Type 4, which is known for its weakness of blood vessels, previous study mentioned that coil embolization via the venous is more appropriate than the arterial route in an attempt to avoid arterial dissection. ${ }^{15)}$ Studies reporting several complications, such as vascular dissection, as a result of catheter insertion in patients with SAM have not been conducted yet, but blister-like aneurysm, which was associated with SAM, has a higher risk of intraoperative rupture and dissection formation than normal aneurysms. Therefore, when treating vascular abnormalities associated with SAM, careful catheterization is required and intravenous embolization may need to be considered. When the fistula is located distally and the microcatheter cannot be introduced into the draining veins, embolization with liquid embolic agents, such as N-butyl-cyanoacrylate, is an effective method. ${ }^{13)}$ However, since MMA has multiple dangerous anastomoses and several branches that nourish the nerve, liquid embolic agents should be injected carefully.

Blood blister-like aneurysm ${ }^{16)}$ and vertebra-vertebral arteriovenous fistula ${ }^{17)}$ have been reported in cases of head and neck angiopathy due to SAM. However, studies assessing the association between MMAVF and SAM have not been conducted yet. SAM causes changes in veins adjacent to arteries, resulting in edematous walls and loss of morphologic definition of the venous media and adventitia, separation, and patchy loss of muscles. ${ }^{18)}$ The MMA runs along the outer surface of the dura accompanied by paired veins. Therefore, when affected by SAM, the MMV may also undergo secondary degeneration, resulting in a fistula. Histologically, it has been reported that the media that is generally denatured in SAM is partially defective at the bifurcation of the intracranial MMA. ${ }^{19}$ ) The association between the characteristics of the partial defect of the intracranial MMA media and the fistula's location remains unclear, and further investigation is required.

Moreover, the fenestration of the right cervical ICA and the fusiform aneurysm of the left cervical ICA might also be associated with SAM. Aneurysms have been reported in cases of head and neck angiopathy due to SAM, ${ }^{16)}$ but the association between cervical ICA fenestration and SAM has not been reported yet. Fenestration, which is defined as a short focal segment of the artery with two patent lumens and congenital fenestration of the cervical ICA, is extremely rare. ${ }^{20,21)}$ There is no clear embryologic mechanism revealing the occurrence of a cervical ICA fenestration. Some previous reports have shown that fenestration was associated with aneurysms, ${ }^{20)}$ and other studies have reported that cervical ICA fenestration was possibly associated with FMD and trauma. ${ }^{21)}$ This case report indicated that cervical ICA fenestration caused by SAM remains possible. 


\section{Conclusion}

In conclusion, MMAVF may be associated with SAM. Closure of the fistula by endovascular means is an effective treatment for MMAVF associated with SAM. In idiopathic MMAVF, intraperitoneal vascular evaluation should be considered to rule out SAM and avoid the potentially fatal complications. SAM can create fistulas between the artery and adjacent vein. ${ }^{18)}$ Therefore, it is important to carefully check for systemic diseases, such as SAM, especially when atypical arteriovenous fistulas are found between an artery and the adjacent vein in any part of the body. Furthermore, as cervical ICA fenestration is possibly associated with SAM, it is assumed that evaluation of systemic diseases, including SAM, is necessary when unusual blood vessel fenestration is observed. Further evaluation of the natural history and origin of idiopathic MMAVF and cervical ICA fenestration is important.

\section{Disclosure Statement}

The authors have no conflicts of interest to declare.

\section{References}

1) Freckmann N, Sartor K, Herrmann HD. Traumatic arteriovenous fistulae of the middle meningeal artery and neighbouring veins or dural sinuses. Acta Neurochir (Wien) 1981; 55: 273-281.

2) Almefty RO, Kalani MY, Ducruet AF, et al. Middle meningeal arteriovenous fistulas: a rare and potentially high-risk dural arteriovenous fistula. Surg Neurol Int 2016; 7: 219-222.

3) Wilson CB, Cronic F. Traumatic arteriovenous fistulas involving middle meningeal vessels. JAMA 1964; 188: 953-957.

4) Chandrashekar HS, Nagarajan K, Srikanth SG, et al. Middle meningeal arteriovenous fistula and its spontaneous closure. A case report and review of the literature. Interv Neuroradiol 2007; 13: 173-178.

5) Takeuchi S, Takasato Y, Masaoka H, et al. A case of traumatic middle meningeal arteriovenous fistula on the side of the head opposite to the injured side. No Shinkei Geka 2009; 37: 983-986.

6) Minami H, Miki T, Kakita H, et al. Traumatic aneurysm of the middle meningeal artery presenting with traumatic middle meningeal arteriovenous fistula: a case report. JNET J Neuroendovasc Ther 2015; 9: 84-89.

7) Kalva SP, Somarouthu B, Jaff MR, et al. Segmental arterial mediolysis: clinical and imaging features at presentation and during follow-up. J Vasc Interv Radiol 2011; 22: 13801387.

8) Baker-LePain JC, Stone DH, Mattis AN, et al. Clinical diagnosis of segmental arterial mediolysis: differentiation from vasculitis and other mimics. Arthritis Care Res (Hoboken) 2010; 62: 1655-1660.

9) Ko M, Kamimura K, Ogawa K, et al. Diagnosis and management of fibromuscular dysplasia and segmental arterial mediolysis in gastroenterology field: a mini-review. World J Gastroenterol 2018; 24: 3637-3649.

10) Satoh T, Sakurai M, Yamamoto $Y$, et al. An interesting case of the traumatic middle meningeal arteriovenous fistula. No Shinkei Geka 1982; 10: 739-745.

11) Slavin RE, Gonzalez-Vitale JC. Segmental mediolytic arteritis: a clinical pathologic study. Lab Invest 1976; 35 : 23-29.

12) Bradac GB, Schramm J, Kaernbach A. Traumatic exophthalmos due to an uncommon arteriovenous fistula without involvement of the cavernous sinus. Case report. J Neurosurg 1981; 55: 993-996.

13) Tsumoto T, Nakakita K, Hayashi S, et al. Bone defect associated with middle meningeal arteriovenous fistula treated by embolization--case report. Neurol Med Chir (Tokyo) 2001; 41: 42-47.

14) Kawaguchi $T$, Kawano $T$, Kaneko $Y$, et al. Traumatic lesions of the bilateral middle meningeal arteries-case report. Neurol Med Chir (Tokyo) 2002; 42: 221-223.

15) Chuman H, Trobe JD, Petty EM, et al. Spontaneous direct carotid-cavernous fistula in Ehlers-Danlos syndrome type IV: two case reports and a review of the literature. $J \mathrm{Neu}$ roophthalmol 2002; 22: 75-81.

16) Tanaka K, Fujiwara M, Okuda Y, et al. A ruptured blood blister-like aneurysm associated with intraperitoneal hemorrhage due to segmental arterial mediolysis: a case report and literature review. World Neurosurg 2020; 134: 79-85.

17) Kuramoto $Y$, Shimo D, Hori S, et al. A case of vertebro-vertebral arteriovenous fistula clinically diagnosed as segmental arterial mediolysis complicated by celiac artery aneurysm suspected in a state of impending rupture. JNET J Neuroendovasc Ther 2015; 9: 84-89.

18) Inada $K$, lkeda $T$, Shimokawa $K$, et al. Venous lesions in segmental arterial mediolysis (SAM). Pathol Clin Med 2005; 23: 1357-1362.

19) Hassler O. Medial defects in the meningeal arteries. $J$ Neurosurg 1962; 19: 337-340.

20) Hasegawa $T$, Kashihara $K$, Ito $H$, et al. Fenestration of the internal carotid artery. Surg Neurol 1985; 23: 391-395.

21) Gailloud P, Carpenter J, Heck DV, et al. Pseudofenestration of the cervical internal carotid artery: a pathologic process that simulates an anatomic variant. AJNR Am J Neuroradiol 2004; 25: 421-424. 